\title{
Illuminant spectrum estimation at a pixel
}

\author{
Sivalogeswaran Ratnasingam ${ }^{1, *}$ and Javier Hernández-Andrés ${ }^{2}$ \\ ${ }^{1}$ Intelligent Systems Research Centre, School of Computing and Intelligent Systems, Magee Campus, University of Ulster, \\ Londonderry, Northern Ireland, BT48 7JL, UK \\ ${ }^{2}$ Department of Optics, Sciences Faculty, University of Granada, Granada, 18071, Spain \\ ${ }^{*}$ Corresponding author: s.ratnasingam@ulster.ac.uk
}

Received February 11, 2011; accepted February 12, 2011;

posted February 16, 2011 (Doc. ID 142491); published March 31, 2011

In this paper, an algorithm is proposed to estimate the spectral power distribution of a light source at a pixel. The first step of the algorithm is forming a two-dimensional illuminant invariant chromaticity space. In estimating the illuminant spectrum, generalized inverse estimation and Wiener estimation methods were applied. The chromaticity space was divided into small grids and a weight matrix was used to estimate the illuminant spectrum illuminating the pixels that fall within a grid. The algorithm was tested using a different number of sensor responses to determine the optimum number of sensors for accurate colorimetric and spectral reproduction. To investigate the performance of the algorithm realistically, the responses were multiplied with Gaussian noise and then quantized to 10 bits. The algorithm was tested with standard and measured data. Based on the results presented, the algorithm can be used with six sensors to obtain a colorimetrically good estimate of the illuminant spectrum at a pixel. (C) 2011 Optical Society of America

OCIS codes: $\quad 330.0330,330.1690,330.1720,330.1730,150.1135,150.6044$.

\section{INTRODUCTION}

The human visual system perceives color of an object largely independent of viewing environment. However, image sensor responses depend on the spectral reflectance of the object, spectral power distribution of the surface, and the geometry of the scene [1]. Consumer cameras use three sensors (red, green, and blue) to capture a scene, and three sensors are necessary and sufficient for colorimetric reproduction [2]. More than three imaging sensors are required to reproduce a scene color representation or reflectance spectra that is sufficiently accurate and independent of both the power spectrum of the light source and imaging device [3].

In recent years, the interest in multispectral image reproduction has increasingly attracted researchers $[4,5]$. The reasons for this increasing interest in spectral reproduction are the advancement in electronic technology, including improvement in processing power and memory, as well as new applications, including electronic archival of art paintings, historical documents, and artificial reillumination of a scene in the film industry. In addition, trichromatic color reproduction suffers from metamerism. For the above reasons, there is an increasing interest in multispectral scene recording. Recently, Ratnasingam et al. [6] investigated the advantages of using the information beyond the visible region for chromaticity estimation. However, using the information beyond the visible region for spectral estimation is dependent on the application. Many researchers concentrated on spectral reproduction with the assumption of either known illuminant spectra or known reflectance spectra $[7,3]$. An algorithm is proposed in this paper to estimate the spectral power distribution of a light source illuminating a scene with no prior knowledge about the scene.

In the past, researchers have used several different methods for spectral estimation, including Wiener estimation [ㄷ,],

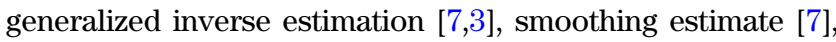
principle component analysis [2], singular value decomposi- tion [8], and linear basis functions [9, 10]. Maloney and Wandell [11] proposed a method for estimating the reflectance spectra using linear basis functions. In estimating the reflectance spectra, they assumed that the spectral sensitivity of the camera and the power spectral distribution of the illuminant are known. Imai and Berns [4] proposed a method for estimating spectral reflectance using a three-sensor camera combined with either absorption filters or multiple illuminants. The estimation matrix is empirically determined using the least squares analysis. The above discussed methods $[\underline{4}, 11]$ used linear basis functions to obtain a possible solution to the reflectance spectrum for a given camera. In both of these methods $[\underline{4}, \underline{11}]$, the dimensionality of the reflectance model is smaller than or equal to the number of different sensors in the camera. Researchers have shown with their results that the optimal number of sensors should match the dimensionality of the reflectance model. As a three-sensor camera is used, the number of linear basis functions is therefore three or less. The required number of basis functions to obtain an acceptable estimate of the spectra is entirely dependent on the type of data sets applied in the test and the number of pigments that were used in creating the reflectance data set $[12,13]$. To use more basis functions in spectral estimation of any data set, Shi and Healey [14] proposed an estimation approach that uses a high-dimensional linear reflectance functions. Most of these techniques assumed that the spectral sensitivities of the imaging device were measured or mathematically derived $[\underline{4}, 14,15]$. For practical use, it is important for being able to estimate the spectra of either the surface reflectance or illuminant power spectrum with prior knowledge of neither the light source used to illuminate the scene nor the spectral reflectance of the object being imaged. In this paper, we propose an algorithm to estimate the illuminant power spectrum at a pixel with no prior knowledge about the reflectance or illuminant. 
A brief description of Ratnasingam and Collins' [ $\underline{16}]$ algorithm and a typical illuminant invariant chromaticity space are given in Section 2. A method for obtaining twodimensional illuminant invariant feature space and the proposed algorithm are described in Section 3. Section 4 presents the results of the algorithm and discussion on the obtained results. Finally, conclusions are made in Section $\underline{5}$.

\section{ILLUMINANT INVARIANT CHROMATICITY SPACE}

An algorithm to extract two illuminant invariant chromaticity features using four sensor responses at pixel level has been proposed by Ratnasingam and Collins [16]. In deriving this algorithm, they assumed that the power spectrum of an illuminant can be approximated by a blackbody spectrum and the imaging sensors sample the scene at a single wavelength. Under these assumptions, it is possible to extract two illuminant invariant features $\left(F_{1}\right.$ and $\left.F_{2}\right)$ from the responses of the four sensors using Eqs. (1) and (2) [ㅌ]:

$$
\begin{aligned}
& F_{1}=\log \left(r_{2}\right)-\left\{\alpha \log \left(r_{1}\right)+(1-\alpha) \log \left(r_{3}\right)\right\}, \\
& F_{2}=\log \left(r_{3}\right)-\left\{\gamma \log \left(r_{2}\right)+(1-\gamma) \log \left(r_{4}\right)\right\},
\end{aligned}
$$

where $r_{1}, r_{2}, r_{3}$, and $r_{4}$ are the responses of the four image sensors and $\alpha$ and $\gamma$ are channel coefficients. The variations caused by both the illuminant intensity and illuminant power spectrum can be removed if the two channel coefficients sa-

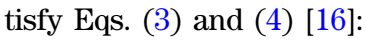

$$
\begin{aligned}
& \frac{1}{\lambda_{2}}=\frac{\alpha}{\lambda_{1}}+\frac{1-\alpha}{\lambda_{3}}, \\
& \frac{1}{\lambda_{3}}=\frac{\gamma}{\lambda_{2}}+\frac{1-\gamma}{\lambda_{4}},
\end{aligned}
$$

where $\lambda_{1}, \lambda_{2}, \lambda_{3}$, and $\lambda_{4}$ are the peak sensor positions.

Similar to other researchers [1,17], Ratnasingam and Collins [16] also assumed that the sensors are infinitely narrow and the illuminant spectrum can be modeled by a blackbody illuminant. However, technologically, it is difficult to make an image sensor that samples the scene at a single wavelength. Equally importantly, sensors with a very narrow spectral response will be starved of photons and hence have a low sensitivity to incoming light. To study this model-based algorithm [16] with real cameras, it is important to determine the effect of using image sensors that respond to a significantly wider range of wavelengths in the visible spectrum. To study the effect of the spectral width on the performance of the model-based algorithm, Ratnasingam and Collins [16] used a Gaussian function to model the spectral sensitivity of the sensors for different spectral widths. To investigate the effect of using a nonblackbody illuminant, Ratnasingam et al. $[\underline{16}, \underline{18}]$ also tested the performance of the algorithm with International Commission on Illumination (CIE) standard daylight and measured daylight. Based on their investigation, the important conclusion made was that the model-based algorithm can be used to extract useful illuminant independent chromaticity features with image sensors of FWHM of

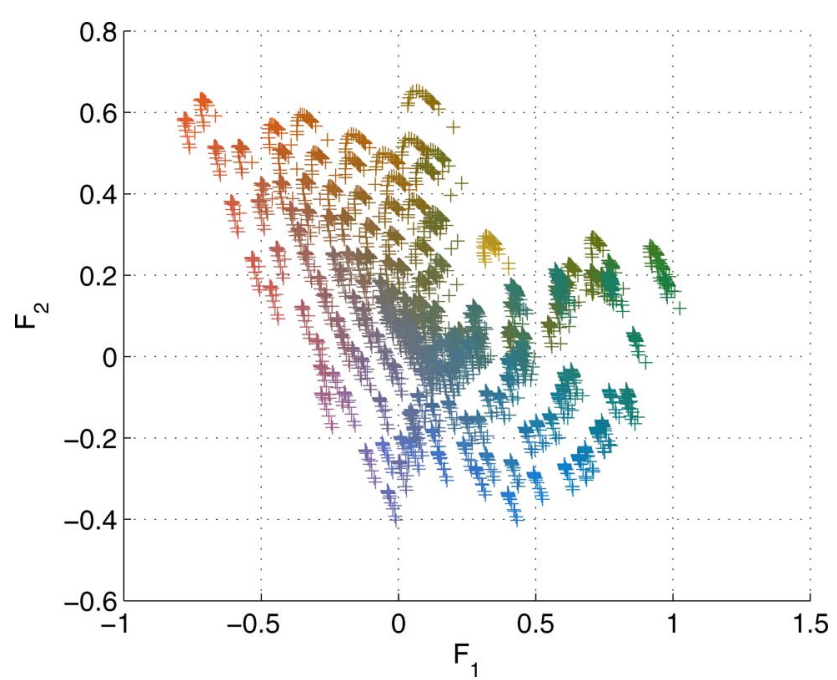

Fig. 1. (Color online) Typical chromaticity space formed by the model-based algorithm with unquantized responses of evenly spread Gaussian sensors of FWHM of $60 \mathrm{~nm}$. In this space, 206 Munsell samples are projected when illuminated by 20 spectra of CIE standard daylights.

$80 \mathrm{~nm}$ or less when illuminating with illuminants, including daylight [16].

A typical feature space formed using the model-based algorithm when applying the responses generated by evenly spread four Gaussian sensors of FWHM of $60 \mathrm{~nm}$ is shown in Fig. 1. The peak sensor positions are 437.5, 512.5, 587.5, and $667.5 \mathrm{~nm}$. Munsell reflectances [19] and CIE standard daylight spectra [20] were used in generating the required image sensor responses. In Fig. 1, 206 Munsell reflectances (samples with CIELab " $L$ " value around 67 ) are projected when illuminated by 20 spectra of CIE standard daylight illuminants. In the illuminant set, the particular correlated color temperatures (CCT) were chosen to represent the distribution of CCT in the measured daylight $[16,21,22]$. Each cross shows the color of a reflectance when illuminated by one of the illuminant spectrum. Close investigation of the space shows that the clusters formed by each of the Munsell reflectances are small in size and perceptually similar reflectances are located as neighboring clusters. This suggests that, by using the two-dimensional space, a rough estimate of the reflectance can be made independent of the light source illuminating the scene. This estimate could be used as the starting point in estimating the illuminant spectrum.

The possibility of accurate spectral reproduction has been investigated with different numbers of sensor responses. In trichromatic reproduction, three sensor responses are used, however, as mentioned in Section $\underline{1}$, trichromatic reproduction suffers from metameric problem. The metameric problem arises when a small number of sensor responses are used to represent a reflectance. To investigate the required number of image sensors to capture a scene to obtain an estimate of the illuminant spectra that is a colorimetrically good match, different numbers of sensors that could be easily accommodated in a consumer camera Bayer pattern has been investigated. As consumer cameras have three sensors, first the possibility of spectral reproduction with three sensors has been investigated. The Bayer pattern has four sensors, so it is relatively easy to accommodate four different sensors in a consumer camera. Therefore, the possibility of using four sensors is investigated as well. It is also possible to make six and eight 
Table 1. Parameters of the Gaussian Sensitivity Functions

\begin{tabular}{ccc}
\hline $\begin{array}{c}\text { Number } \\
\text { of Sensors }\end{array}$ & FWHM (nm) & $\begin{array}{c}\text { Peak Sensor } \\
\text { Positions (nm) }\end{array}$ \\
\hline 3 & 80 & $450,550,650$ \\
4 & 80 & $437,512,587,637$ \\
6 & 80 & $425,475,525,575,625,675$ \\
8 & 60 & $419,456,494,531,569,606,644,681$ \\
\hline
\end{tabular}

sensors by taking two blocks of Bayer pattern to form a single pixel. Based on this discussion, three, four, six, and eight sensors were used in this investigation. Table 1 lists the parameters of the image sensors used to calculate the responses. In this initial investigation, the particular peak sensor positions were chosen in such a way that the sensitivity functions are evenly spread across the entire visible spectrum (400 to $700 \mathrm{~nm}$ ). This is important to capture the scene data equally in all parts of the visible range [16]. Ratnasingam and Collins [16] have shown that their model-based algorithm can extract useful illuminant invariant features with an image sensor width of $80 \mathrm{~nm}$ or less. As the consumer cameras such as Sony DXC930 cameras have sensor spectral width of approximately $80 \mathrm{~nm}$, to investigate the proposed algorithm with feasible sensor width, $80 \mathrm{~nm}$ FWHM was chosen. However, when the number of sensors increases, the overlap between sensitivity functions increases, which leads to increases in noise effect [16]. To avoid this significant overlap between sensitivity functions, a $60 \mathrm{~nm}$ FWHM Gaussian function was used when applying eight sensors.

\section{ALGORITHM}

The first step of spectral estimation is forming a twodimensional illuminant invariant feature space. As can be seen from Eqs. (1) and (2), forming two illuminant invariant features using the method described by Ratnasingam and Collins [16] requires a minimum of four different sensor responses. Therefore, to form a two-dimensional feature space from three sensor responses, a different approach has been described. Consider the basic image equation for an imaging sensor with sensitivity function $c(\lambda)$, sensor response $r^{x, E}$ is given by [1]

$$
r^{x, E}={\underset{-}{a}}_{-}^{x} \cdot n^{x} I^{x} \int_{400 \mathrm{~nm}}^{700 \mathrm{~nm}} S^{x}(\lambda) e(\lambda) c(\lambda) \mathrm{d} \lambda,
$$

where $e(\lambda)$ is the power spectrum of the illuminant and $s(\lambda)$ is the reflectance spectrum of the object. The dot product $a^{x} \cdot n^{x}$ models the geometry factor of a scene. Unit vectors $\alpha^{x}$ and $\bar{n}^{x}$ represent the direction of light source and the direction of surface normal, respectively. The term $I^{x}$ is the power of the incident light at point $x$ on the scene. The integration is over the visible range.

Equation (5) can be simplified by assuming that the spectral sensitivity function can be approximated by a Dirac delta function. The sifting property of the Dirac delta function can then be applied to simplify Eq. (5), therefore, a sensor that is effectively only sensitive to light at a single wavelength $\lambda_{i}$ becomes

$$
r^{x, E}=\underline{a}_{-}^{x} \cdot n^{x} I^{x} s^{x}\left(\lambda_{i}\right) e\left(\lambda_{i}\right)
$$

The different components of the above equation can be separated by taking the logarithm to both sides:

$$
\log \left(r^{x, E}\right)=\log \left\{g I^{x}\right\}+\log \left\{e\left(\lambda_{i}\right)\right\}+\log \left\{s^{x}\left(\lambda_{i}\right)\right\},
$$

where $g\left(=a^{x} \cdot n^{x}\right)$ is the geometry factor.

From Eq. (ㄱ) , it can be seen that taking the logarithm difference between two sensor responses removes the geometryand illuminant intensity-dependent components. It is known that, in daylight scenes, the intensity varies significantly compared to the chromaticity of the illuminant. Therefore, taking the features formed by the logarithm difference between two sensor responses could be used to form two features with three sensor responses. The equations for both features are

$$
\begin{aligned}
& F_{1}=r_{3}-r_{2}, \\
& F_{2}=r_{3}-r_{1},
\end{aligned}
$$

where $r_{1}, r_{2}$, and $r_{3}$ are the image sensor responses. The sensors are numbered starting from the shortest wavelength end. In this investigation, evenly spread sensors in the visible spectrum ( 400 to $700 \mathrm{~nm}$ ) were used and the spectral sensitivity of the sensors was modeled using the Gaussian function. According to Ratnasingam and Collins [16], there are four possible features that can be formed from six sensor responses. From these features, two were chosen in such a way that the color variation across the feature space is smooth and the correlation between the features is the minimum. The particular sensor responses chosen in generating the features are the sensor responses $r_{1}, r_{3}$, and $r_{5}$ to form feature 1 and $r_{2}, r_{4}$, and $r_{6}$ to form feature 2 to obtain the two-dimensional illuminant invariant space. Similarly, for the eight-sensor case, sensors $r_{1}, r_{4}$, and $r_{7}$ and sensors $r_{2}, r_{5}$, and $r_{8}$ were used to form the two illuminant invariant features. The illuminant invariant two-dimensional feature space was used to estimate the illuminant power spectrum using a generalized inverse estimate [7].

In the past, researchers have used several different methods for spectral estimation. The performance of these methods in estimating the spectrum is comparable [23]. Among those methods, the generalized inverse estimate is less complex and therefore requires less processing power. Consider the discrete form of image Eq. (5) for an image sensor

$$
r=\sum_{i=400}^{700} I g s_{i} e_{i} c_{i}
$$

For a set of $p$ image sensors sampling the scene at $q$ different wavelengths, the matrix form of the image equation can be given by

$$
R=I g C S E
$$

where $R$ is the px1 vector of sensor responses, $C$ is a pxq matrix of a set of spectral sensitivities, $S$ is the qxq diagonal matrix of reflectance spectra, and $E$ is a qx1 vector of illuminant power spectrum. For abbreviation, let $W_{I}=I g C S$, the estimated power spectrum of an illuminant can be given by the generalized inverse estimate [7]: 


$$
\hat{E}=W_{I}^{-1} R=W_{I}^{T}\left(W_{I} W_{I}^{T}\right)^{-1} R .
$$

Applying this equation to estimate the illuminant power spectrum with no prior knowledge about the scene (i.e., reflectance being imaged) resulted in a very poor estimate. To obtain a better estimate of the illuminant power spectrum, the two-dimensional illuminant invariant feature space described in Section 2 was used. In Eq. (12), for a given camera, the weight matrix $\left(W_{I}\right)$ is dependent on the reflectance spectra of the surface being imaged. A close investigation of the twodimensional feature space (see Fig. 1) shows a smooth perceptual variation across the space. To take advantage of the illuminant invariant feature space in estimating the illuminant spectrum, the feature space was divided into uniform rectangular grids $(50 \times 50)$ in such a way that the reflectances that fall within a grid are perceptually similar. The matrix $W_{I}$ was calculated for each of these grids using the training reflectance set and illuminant spectra. In the test phase for a particular test illuminant and test reflectance, the two illuminant invariant features were projected onto the two-dimensional feature space, and the corresponding $W_{I}$ matrix, calculated for that particular grid during training, was used to estimate the power spectrum of the illuminant that was used to illuminate the test reflectance.

For comparison of the estimation methods, the Wiener estimation method was also applied instead of generalized inverse estimation. The same procedure described above was followed to estimate the illuminant power spectrum using the Wiener estimation method. The Wiener estimation can be given by $[\underline{7,}, \underline{8}]$

$$
\hat{E}=E_{s s} W_{w}^{T}\left(W_{w} E_{s s} W_{w}^{T}\right)^{-1} R,
$$

where $E_{s s}$ is the autocorrelation matrix of the illuminant power spectrum and $W_{w}$ is the weight matrix for Wiener estimation.

\section{RESULTS AND DISCUSSION}

To obtain conclusions independent of the data set, the performance of the algorithm was investigated with commonly used Munsell reflectances [19], Floral reflectances [24], CIE standard daylight [20], and measured daylight [21]. From each of these reflectance data, a set of 20 reflectance spectra were randomly chosen as a training set and another 20 set for testing the algorithm. From the CIE standard daylight, 20 spectra were chosen for training and another 20 spectra were chosen for testing the algorithm. In both of these CIE standard daylight sets, particular CCT values (between 4000 and 25, $000 \mathrm{~K}$ ) were chosen in such a way that the distribution of the CCT is similar to the measured daylight $[\underline{16}, 21,22]$. From the measured daylight, 20 spectra were chosen from the first year (1996) and another 20 were chosen from the second year (1997) measurements for testing the algorithm. Both of these measured daylight sets were chosen in such a way that they were measured throughout the year at different times of day. To investigate the algorithm accurately, all the data was sampled at $1 \mathrm{~nm}$ intervals. The sensitivity of image sensors were modeled by evenly spread Gaussian functions. The image sensor responses were calculated by numerically integrating the basic image Eq. (5). As it has been shown that the CIE standard daylight is not a good representative model for the measured daylight [18], the weight matrix $W$ was calculated separately for CIE standard daylight and measured daylight.

To assess the performance of the algorithm quantitatively, it is required to use a metric that measures the spectral match between the actual and the estimated spectra. A well-known measure that has been widely used to measure the accuracy of the spectra is the goodness-fitting coefficient (GFC) [25]. As a colorimetric interpretation of GFC it is generally defined as follows: GFC $>0.999$ is very good match, GFC $\geq 0.99$ is a good match, and a GFC around 0.99 is a satisfactory reproduction [2].

To investigate the colorimetric performance of an algorithm, two uniform color spaces (CIELab and CIELuv) have been defined by the CIE. Generally, in assessing the color match between two self-luminous objects CIELuv color space is used [26]. As the CIELuv space is a perceptually uniform space, the perceptual difference can be related to the geometric distance in the space. In CIELuv color space, three to five Euclidean distance is often taken as just noticeable difference [21]. Abrardo et al. [27] classified that the difference of $0-1$ as limit of perception, $1-3$ as very good match, $3-6$ as good match, $6-10$ as sufficient match, and more than 10 as insufficient quality. In the CIELuv space, $L=100$ is bright white and $L=0$ is absolute black. In calculating the color difference between the actual and the estimated spectra, both were scaled in such a way that the $L$ coordinate of both spectra is 50 units. This $L=50$ plane was used as the reference plane in defining the CIE standard perceptual color difference model [3]].

Figures 2-5 show typical results when applying the generalized inverse estimation and the Wiener estimation to obtain an estimate of the illuminant spectrum with different numbers of sensor responses. These figures show the spectrum of CIE standard daylight D65 and the estimated spectrum using the proposed algorithm using both estimation methods. In these initial simulations, noise was not included in the sensor responses. As can be seen when the number of input sensor responses increases, a better estimate of the spectra was obtained. Close investigation of Fig. 2 shows that using three sensor responses does not give a good estimate of the spectrum. However, using four sensor responses (see Fig. 3) results in an estimate that approximates the overall shape of the illuminant power spectrum. As the number of input sensor responses increases, the estimated spectrum approximates the local structures in the illuminant spectra caused by the atmospheric attenuation (see Fig. 5). Comparing the estimates obtained using both estimation methods, the generalized inverse estimation gives a better estimate for a small number of sensors (up to six sensors, see Figs. 2-4). However, Wiener estimation gives a better estimate with a larger number of sensors (see Fig. 5). Particularly, the Wiener estimation method estimates the wiggles caused by the atmospheric animation when using eight image sensor responses.

The proposed algorithm was tested with standard (Munsell, CIE daylight) and measured (floral and measured daylight) test data sets. Based on the above discussion, two measures (GFC and CIELuv color difference) were used to determine the goodness of the estimated spectra. For both measures, the mean value and the standard deviation were calculated. Test results of the algorithm using generalized inverse estimation and the Wiener estimation when applying unquantized sensor responses with zero Gaussian noise are listed in 


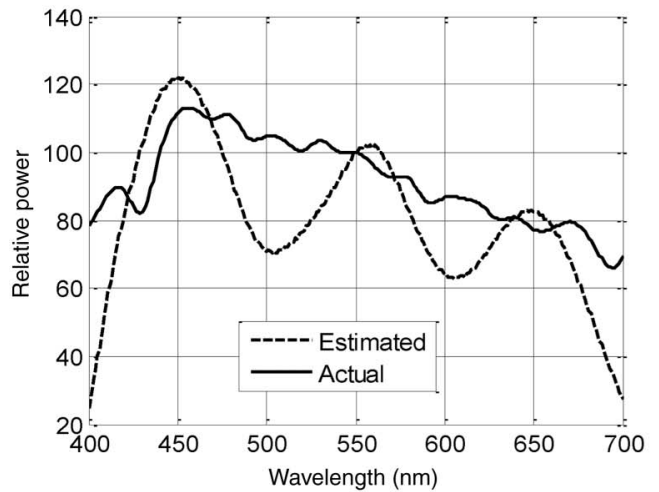

(a) Generalized inverse estimation

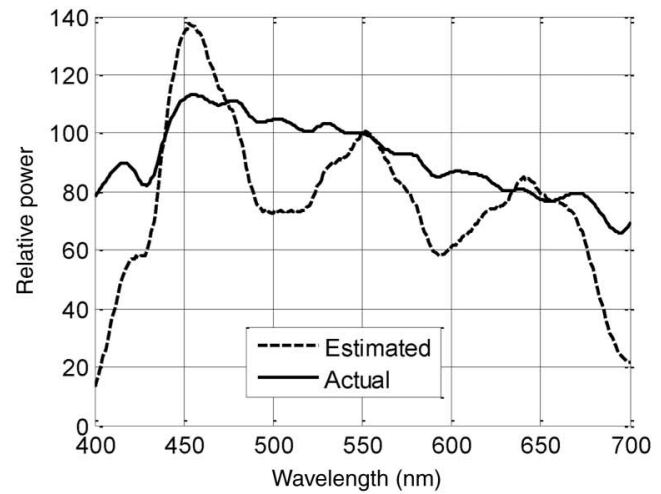

(b) Wiener estimation

Fig. 2. (a) Generalized inverse estimation, (b) Wiener estimation. Actual and estimated illuminant spectra when using three sensor responses. Both spectra are normalized at $550 \mathrm{~nm}$. The CIELuv difference and GFC of the two spectra shown in (a) are 7.04 units and 0.9818 and 8.105 units and 0.9723 for that shown in (b), respectively.

Tables $\underline{2}$ and $\underline{3}$ respectively. From the results listed in Tables $\underline{2}$ and 3 , it can be seen that the algorithm gives a good estimate of the illuminant spectrum when tested with six and eight image sensor responses. Particularly, the proposed algorithm gives a colorimetrically very good estimation when applying eight sensor responses. As the number of input image sensor responses increases, the performance also improves. The reason for this performance improvement is the increase in available information about the scene. However, the improvement in performance of the algorithm is relatively small when increasing the number of sensors from six to eight. The reason could be that six sensors are good enough to obtain a colorimetrically good match. It can also be seen that, as the number of sensors increases, the standard deviation of both GFC and colorimetric distance reduces. A noticeable observation is that, in both estimation methods, the performance of the algorithm that could be achieved with real-world measured daylight is slightly under estimated by CIE standard daylight. Comparing the performance obtained with both estimation methods, it can be seen that the inverse estimation method gives a better performance compared to the Wiener estimation with few sensor responses (three or four sensors). However, when increasing the number of sensor responses (eight sensor responses), the Wiener estimation method gives better performance compared to the inverse estimation method. It can also be seen that both the measures (GFC and CIELuv

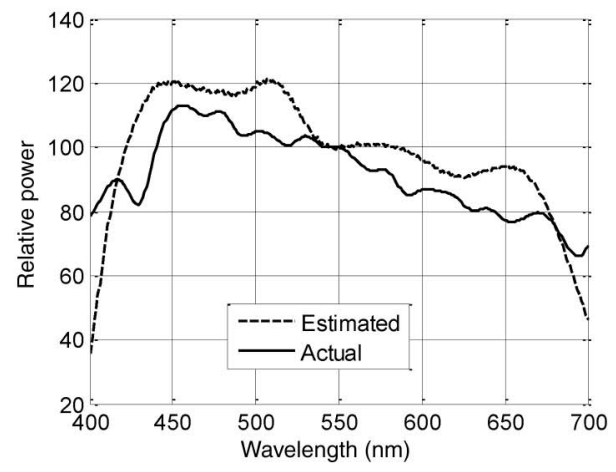

(a) Generalized inverse estimation distance) show a slight difference. As far as the relative shape of the spectrum is concerned, GFC is a more appropriate measure. To overcome the brightness variation between the spectra when calculating the CIELuv distance, the spectra were scaled in such a way that both spectra have the same $L$ coordinate $(L=50)$.

To investigate the performance of the proposed algorithm more realistically, the input to the algorithm was mixed with sensor noise and quantization noise. To take into account the additive and multiplicative noise in a system, quantization noise was modeled as additive noise (not dependent on the magnitude of the sensor response). Sensor noise is generally modeled as a multiplicative noise [10,28]. Based on this evidence, sensor noise was modeled as multiplicative noise. The performance of the proposed algorithm was tested with different levels of sensor noise. The signal-to-noise ratio (SNR) of the data available from a camera depends on several factors, including photon shot noise, the charge storage capacity of the pixel, and the noise introduced by the readout electronics. Winkler and Susstrunk's [29] empirical results show that imagers are available with an SNR larger than $40 \mathrm{~dB}$. Based on this evidence, the performance of the proposed algorithm was investigated with a SNR of $40 \mathrm{~dB}$. The sensor noise was simulated by generating normally distributed random numbers (100 samples) with a mean value of 1 and standard deviations of $1 \%$. The final step in the simulation was to

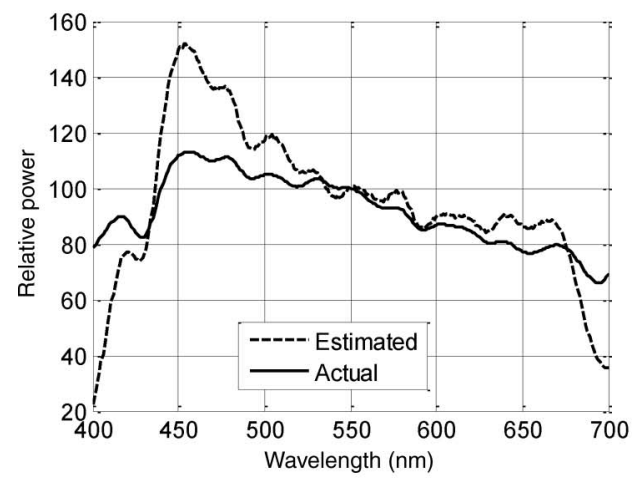

(b) Wiener estimation

Fig. 3. (a) Generalized inverse estimation, (b) Wiener estimation. Actual and estimated illuminant spectra when using four sensor responses. Both spectra are normalized at $550 \mathrm{~nm}$. The CIELuv difference and GFC of the two spectra shown in (a) are 2.35 units and 0.9946 and 6.926 units and 0.9862 for that shown in (b), respectively. 


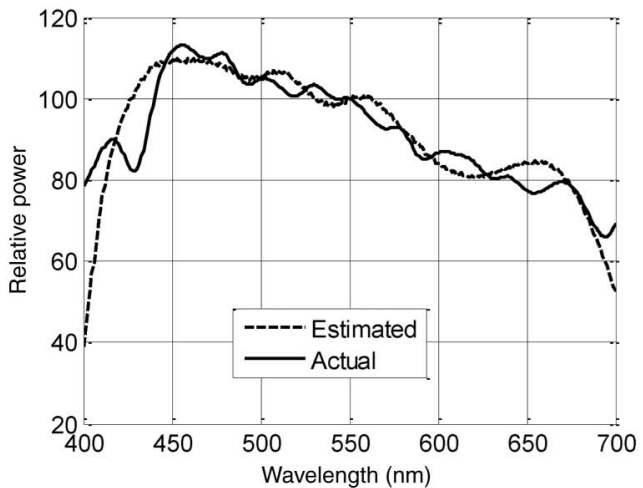

(a) Generalized inverse estimation

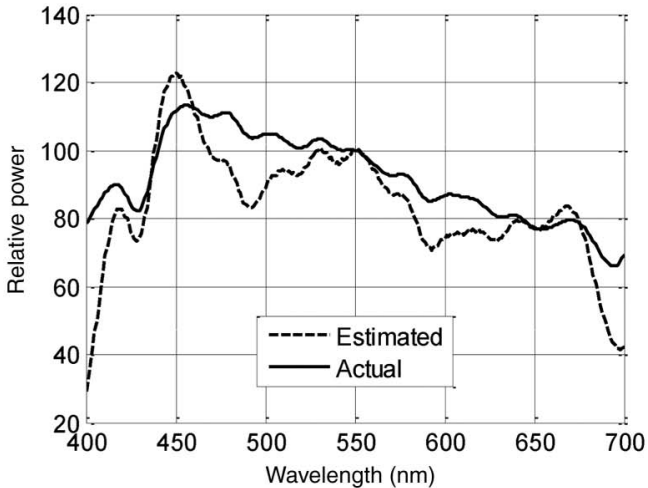

(b) Wiener estimation

Fig. 4. (a) Generalized inverse estimation, (b) Wiener estimation. Actual and estimated illuminant spectra when using six sensor responses. Both spectra are normalized at $550 \mathrm{~nm}$. The CIELuv difference and GFC of the two spectra shown in (a) are 0.987 units and 0.9968 and 2.235 units and 0.9935 for that shown in (b), respectively.

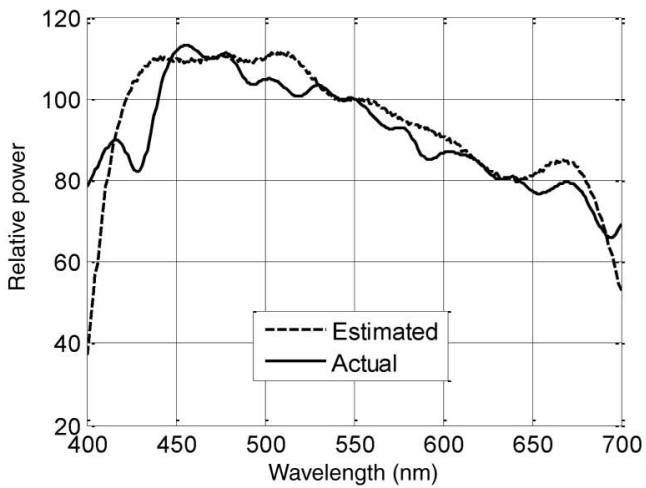

(a) Generalized inverse estimation

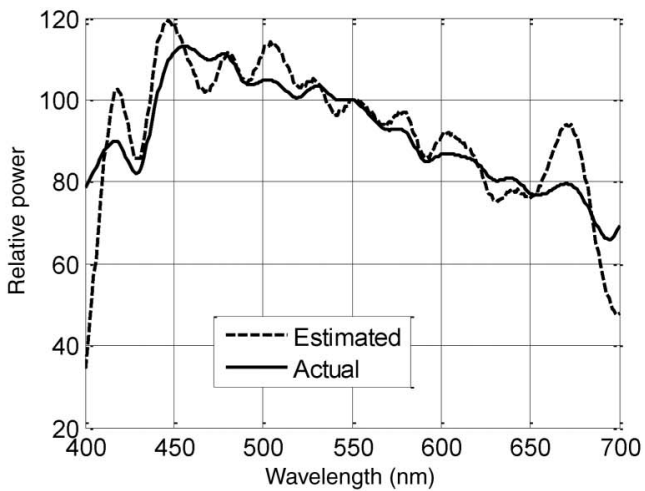

(b) Wiener estimation

Fig. 5. (a) Generalized inverse estimation, (b) Wiener estimation. Actual and estimated illuminant spectra when using eight sensor responses. Both spectra are normalized at $550 \mathrm{~nm}$. The CIELuv difference and GFC of the two spectra shown in (a) are 0.702 units and 0.9965 and 0.4749 units and 0.9961 for that shown in (b), respectively.

Table 2. Test Results of the Algorithm Using Generalized Inverse Estimation When Applying Zero Noise and Unquantized Image Sensor Responses ${ }^{a}$

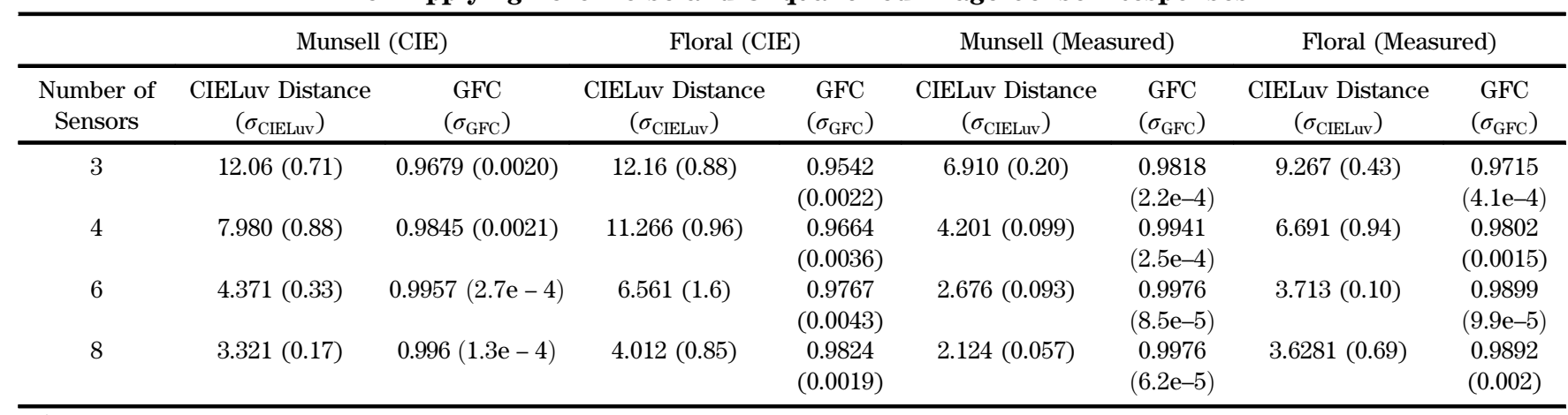

\footnotetext{
${ }^{a}$ Mean Euclidean distance in the CIELuv space between the actual and the estimated spectra, standard deviation $\left(\sigma_{\mathrm{CIELuv}}\right)$, mean GFC, and standard deviation of GFC
} $\left(\sigma_{\mathrm{GFC}}\right)$ are listed. In this test, Munsell floral test reflectances were illuminated by CIE standard and measured test daylights.

represent the effects of using a quantizer to represent the image sensor responses to digital quantities. The effect of quantization was simulated as described by Ratnasingam and Collins [ㄷ]. As cameras are available with a 10 bits analogto-digital converter, in this investigation, sensor responses quantized to 10 bits were used as inputs to the algorithm.

From the results presented in Tables $2-\underline{5}$, it can be seen that the overall performance of the algorithm degrades when the noise level increases. The reason is that, as the noise level increases, the variation in the sensor responses increases. This increase in variation leads the responses to fall in the wrong grid and results in an incorrect estimation of the illuminant power spectrum. In this study, a sensible choice of sensors was used to investigate the performance of the algorithm. However, the performance of the algorithm could be improved by optimizing the sensitivity functions (peak sensor 
Table 3. Test Results of the Algorithm Using Wiener Estimation When Applying Zero Noise and Unquantized Image Sensor Responses ${ }^{a}$

\begin{tabular}{ccccccccc}
\hline & \multicolumn{2}{c}{ Munsell (CIE) } & \multicolumn{2}{c}{ Floral (CIE) } & \multicolumn{2}{c}{ Munsell (Measured) } & \multicolumn{2}{c}{ Floral (Measured) } \\
\hline $\begin{array}{c}\text { Number of } \\
\text { Sensors }\end{array}$ & $\begin{array}{c}\text { CIELuv Distance } \\
\left(\sigma_{\mathrm{CIELuv}}\right)\end{array}$ & $\begin{array}{c}\text { GFC } \\
\left(\sigma_{\mathrm{GFC}}\right)\end{array}$ & $\begin{array}{c}\text { CIELuv Distance } \\
\left(\sigma_{\mathrm{CIELuv}}\right)\end{array}$ & $\begin{array}{c}\text { GFC } \\
\left(\sigma_{\mathrm{GFC}}\right)\end{array}$ & $\begin{array}{c}\text { CIELuv Distance } \\
\left(\sigma_{\mathrm{CIELuv}}\right)\end{array}$ & $\begin{array}{c}\text { GFC } \\
\left(\sigma_{\mathrm{GFC}}\right)\end{array}$ & $\begin{array}{c}\text { CIELuv Distance } \\
\left(\sigma_{\mathrm{CIELuv}}\right)\end{array}$ & $\begin{array}{c}\text { GFC } \\
\left(\sigma_{\mathrm{GFC}}\right)\end{array}$ \\
\hline 3 & $13.4(0.68)$ & 0.9573 & $17.1(1.0)$ & 0.9441 & $13.3(0.61)$ & 0.9587 & $13.2(0.59)$ & 0.9600 \\
& & $(0.0020)$ & & $(0.0031)$ & & $(0.0011)$ & $(0.0013)$ \\
4 & $12.9(1.0)$ & 0.9667 & $14.0(1.8)$ & 0.9528 & $6.91(0.22)$ & 0.9883 & $8.78(1.2)$ & 0.9772 \\
& & $(0.0026)$ & & $(0.0071)$ & & $(1.2 \mathrm{e}-4)$ & $(0.0021)$ \\
6 & $5.52(0.28)$ & 0.9862 & $7.19(1.7)$ & 0.9776 & $3.54(0.097)$ & 0.9946 & $2.90(0.65)$ & 0.9888 \\
& & $(9.5 \mathrm{e}-4)$ & & $(0.0048)$ & & $(1.3 \mathrm{e}-4)$ & $(0.0014)$ \\
8 & $3.27(0.15)$ & 0.9943 & $3.38(1.9)$ & 0.9845 & $1.99(0.055)$ & 0.9971 & $1.20(0.11)$ & 0.9917 \\
& & $(3.9 \mathrm{e}-4)$ & & $(0.0063)$ & & $(6.1 \mathrm{e}-5)$ & $(8.4 \mathrm{e}-5)$ \\
\hline
\end{tabular}

${ }^{a}$ Mean Euclidean distance in CIELuv space between the actual and the estimated spectra, standard deviation $\left(\sigma_{\mathrm{CIELuv}}\right)$, mean GFC, and standard deviation of GFC $\left(\sigma_{\mathrm{GFC}}\right)$ are listed. In this test, Munsell floral test reflectances were illuminated by CIE standard and measured test daylights.

Table 4. Test Results of the Algorithm Using Generalized Inverse Estimation When Applying Different Numbers of Image Sensor Responses ${ }^{a}$

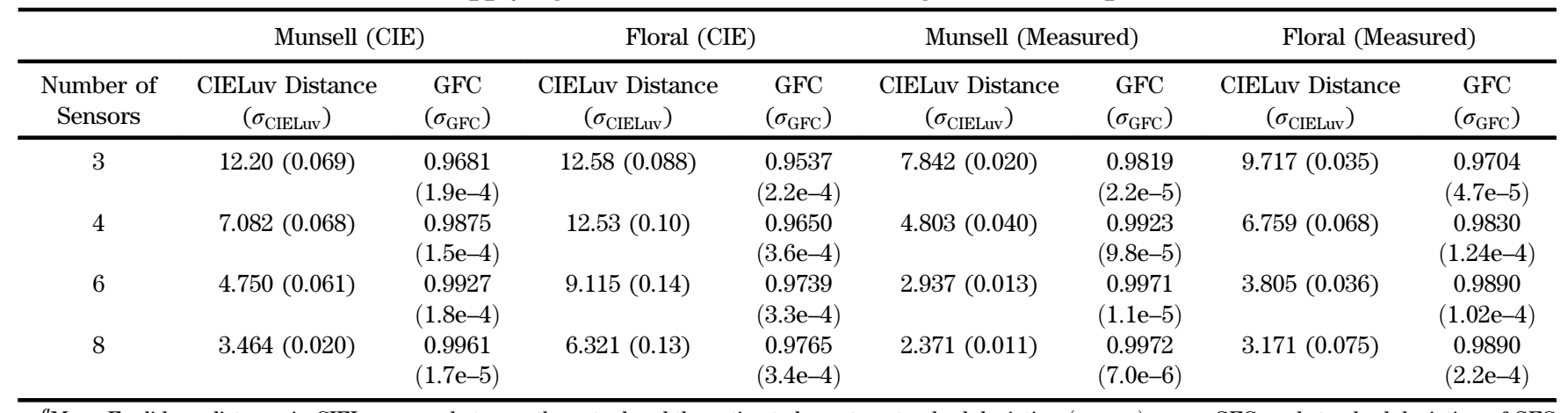

${ }^{a}$ Mean Euclidean distance in CIELuv space between the actual and the estimated spectra, standard deviation $\left(\sigma_{\mathrm{CIELuv}}\right)$, mean GFC, and standard deviation of GFC $\left(\sigma_{\mathrm{GFC}}\right)$ are listed. The sensor responses were multiplied with $40 \mathrm{~dB}$ Gaussian noise, and the resultant responses were quantized to 10 bits. In this test, Munsell floral test reflectances were illuminated by CIE standard and measured test daylights.

Table 5. Test Results of the Algorithm Using Wiener Estimation When Applying Different Numbers of Image Sensor Responses ${ }^{a}$

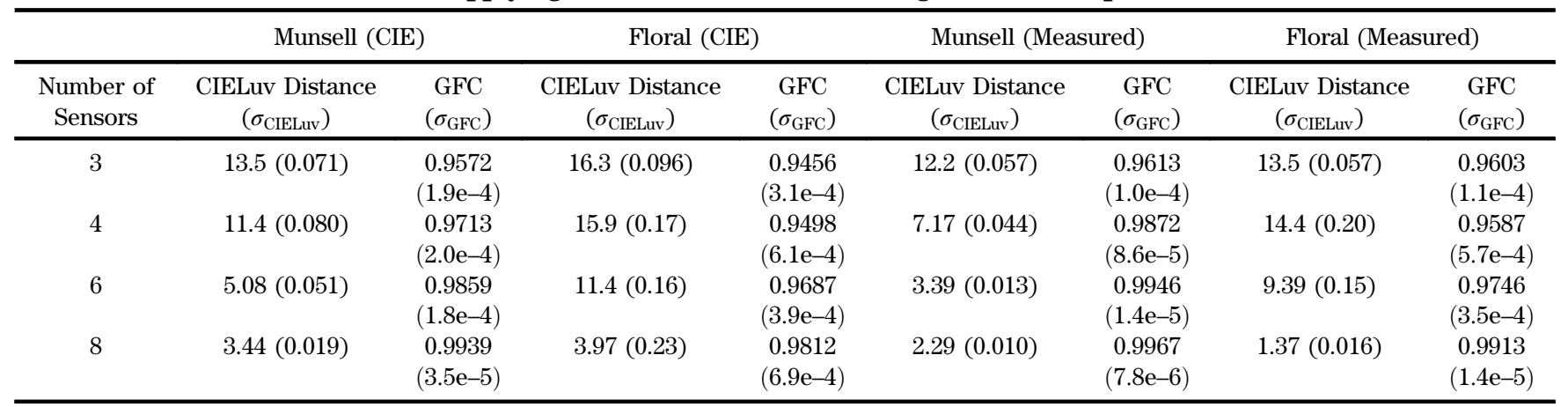

\footnotetext{
${ }^{a}$ Mean Euclidean distance in CIELuv space between the actual and the estimated spectra, standard deviation $\left(\sigma_{\mathrm{CIELuv}}\right)$, mean GFC, and standard deviation of GFC $\left(\sigma_{\mathrm{GFC}}\right)$ are listed. The sensor responses were multiplied with $40 \mathrm{~dB}$ Gaussian noise, and the resultant responses were quantized to 10 bits. In this test, Munsell floral test reflectances were illuminated by CIE standard and measured test daylights.
}

positions and spectral width) of the imaging sensors for a different number of sensors separately. Optimization of the sensitivity functions and investigating the performance of the algorithm with indoor illuminants will be our future work. Based on the results presented in this paper, it can be concluded that the algorithm can be used with six or more sensor responses with a 10 bits quantizer and $40 \mathrm{~dB}$ SNR to obtain a colorimetrically good estimate of the illuminant spectrum at the pixel level.

\section{CONCLUSIONS}

An algorithm has been proposed to estimate the power spectrum of a light source at the pixel level. As the algorithm estimates the illuminant power spectrum at a pixel, it can be used in scenes illuminated by nonuniform illuminants. The algorithm starts with forming a two-dimensional illuminant invariant chromaticity feature space. This feature space was made into small grids in such a way that the reflectances that fall within a grid are colorimetrically similar. A weight matrix 
was calculated for each grid, and the generalized inverse estimation and Wiener estimation methods were applied to estimate the illuminant spectrum of the pixels that fall within that grid. Goodness of the estimated illuminant power spectrum was evaluated with GFC and CIELuv color difference. To evaluate the performance of the algorithm realistically, sensor noise and the effect of quantization were taken into account. The algorithm was tested with a different number of sensor responses to find the optimum number of sensors required to obtain a colorimetrically good estimate of the illuminant spectrum. Based on the results presented, it can be concluded that six sensors are enough to obtain a colorimetrically good estimate of the illuminant spectrum at a pixel with sensor responses of $40 \mathrm{~dB}$ and an SNR quantized to 10 bits.

\section{ACKNOWLEDGMENTS}

This research has been supported by the Centre of Excellence in Intelligent Systems (CoEIS) project, funded by Northern Ireland Integrated Development Fund and InvestNI.

\section{REFERENCES}

1. G. D. Finlayson and S. D. Hordley, "Color constancy at a pixel," J. Opt. Soc. Am. A 18, 253-264 (2001).

2. A. Mansouri, T. Sliwa, J. Y. Hardeberg, and Y. Voisin, "An adaptive-pca algorithm for reflectance estimation from color images," in Proceedings of the 19th IEEE International Conference on Pattern Recognition (IEEE, 2008), pp. 1-4.

3. J. Y. Hardeberg, "Acquisition and reproduction of color images: colorimetric and multispectral approaches," Ph.D. dissertation (Ecole Nationale Supérieure des Télécommunications, 1999).

4. F. H. Imai and R. S. Berns, "Spectral estimation using trichromatic digital cameras," in Proceedings of the International Symposium on Multispectral Imaging and Color Reproduction, (Society of Multispectral Imaging of Japan, 1999), pp. 42-49.

5. H. Haneishi, T. Hasegawa, A. Hosoi, Y. Yokoyama, N. Tsumura, and Y. Miyake, "System design for accurately estimating the spectral reflectance of art paintings," Appl. Opt. 39, 6621-6632 (2000).

6. S. Ratnasingam, S. Collins, and J. Hernández-Andrés, "Extending "color constancy" outside the visible region," J. Opt. Soc. Am. A 28, 541-547 (2011).

7. W. K. Pratt and C. E. Mancill, "Spectral estimation techniques for the spectral calibration of a color image scanner," Appl. Opt. 15 73-75 (1976).

8. N. Shimano, "Optimization of spectral sensitivities with Gaussian distribution functions for a color image acquisition device in the presence of noise," Opt. Eng. 45, 013201 (2006).

9. J. Romero, A. García-Beltrán, and J. Hernández-Andrés, "Linear bases for representation of natural and artificial illuminants," J. Opt. Soc. Am. A 14, 1007-1014 (1997).
10. L. T. Maloney, "Evaluation of linear models of surface spectral reflectance with a small number of parameters," J. Opt. Soc. Am. A 3, 1673-1683 (1986).

11. L. T. Maloney and B. A. Wandell, "Color constancy: a method for recovering surface spectral reflectance,” J. Opt. Soc. Am. A 3, 29-33 (1986).

12. M. J. Vhrel, R. Gershon, and L. S. Iwan, "Measurement and analysis of object reflectance spectra," Color Res. Appl. 19, 4-9 (1994).

13. T. Harifi, S. H. Amirshahi, and F. Agahian., "Recovery of reflectance spectra from colorimetric data using principal component analysis embedded regression technique," Opt. Rev. 15, 302-308 (2008).

14. M. Shi and G. Healey, "Using reflectance models for color scanner calibration," J. Opt. Soc. Am. A 19, 645-656 (2002).

15. J. M. Dicarlo and B. A. Wandell, "Spectral estimation theory: beyond linear but before Bayesian," J. Opt. Soc. Am. A 20, 1261-1270 (2003).

16. S. Ratnasingam and S. Collins, "Study of the photodetector characteristics of a camera for color constancy in natural scenes," J. Opt. Soc. Am. A 27, 286-294 (2010).

17. J. A. Marchant and C. M. Onyango, "Shadow-invariant classification for scenes illuminated by daylight," J. Opt. Soc. Am. A 17, 1952-1961 (2000).

18. S. Ratnasingam, S. Collins, and J. Hernández-Andrés, "Optimum sensors for color constancy in scenes illuminated by daylight," J. Opt. Soc. Am. A 27, 2198-2207 (2010).

19. Munsell Color Science Laboratory, "Daylight spectra," http:// www.cis.rit.edu/mcsl/

20. "Munnsell Colors Matt database," ftp://ftp. cs. joensuu. fi/pub/ color/spectra/mspec/.

21. J. Hernández-Andrés, J. Romero, J. L. Nieves, and R. L. Lee, Jr., "Color and spectral analysis of daylight in southern Europe," J. Opt. Soc. Am. A 18, 1325-1335 (2001).

22. D. B. Judd, D. L. MacAdam, and G. Wyszecki, "Spectral distribution of typical daylight as a function of correlated color temperature," J. Opt. Soc. Am. A 54, 1031-1040 (1964).

23. M. A. López-Álvarez, J. Hernández-Andrés, E. M. Valero, and J. Romero, "Selecting algorithms, sensors and linear bases for optimum spectral recovery of skylight," J. Opt. Soc. Am. A 24, 942-956 (2007).

24. S. E. J. Arnold, V. Savolainen, and L. Chittka, "FReD: the floral reflectance spectra database," Nature Precedings (2008). http:// dx.doi.org/10.1038/npre.2008.1846.1.

25. J. Hernández-Andrés, J. Romero, and R. L. Lee, Jr., "Colorimetric and spectroradiometric characteristics of narrow-field-of-view clear skylight in Granada, Spain,” J. Opt. Soc. Am. A 18, 412-420 (2001).

26. M. Ebner, Color Constancy (Wiley, 2007).

27. A. Abrardo, V. Cappellini, M. Cappellini, and A. Mecocci. "Artworks color calibration using the VASARI scanner," in Fourth Color Imaging Conference: Color Science, Systems, and Applications 4 (The Society for Imaging Science and Technology, 1996), pp. 94-97.

28. G. Sharma and H. J. Trussell, "Digital color imaging," IEEE Trans. Image Process. 6, 901-932 (1997).

29. S. Winkler and S. Susstrunk, "Visibility of noise in natural images," Proc. SPIE 5292, 121-129 (2004). 